\title{
Vitmamin D supplementation with Cardiac Rehabilitation reduces the number of RVOT Premature Ventricular Contractions
}

\author{
CISMARU Gabriel ${ }^{1}$, GURZAU Diana ${ }^{1}$, FRINGU Florina ${ }^{1}$, MARTIS Alexandru ${ }^{1}$, CALOIAN \\ Bogdan ${ }^{1}$, COMSA Horatiu ${ }^{1}$, GUSETU Gabriel ${ }^{1}$, PUIU Mihai ${ }^{1}$, ISTRATOAIE Sabina ${ }^{3}$, CISMARU \\ Andrei ${ }^{2}$, ROSU Radu ${ }^{1}$, POP Dana ${ }^{1}$, ZDRENGHEA Dumitru ${ }^{1}$
}

Corresponding author: Gabriel CISMARU, E-mail: gabi cismaru@yahoo.com

1. 5th Department of Internal Medicine, Cardiac Rehabilitation, "Iuliu

Hatieganu" University of Medicine and Pharmacy, Cluj-Napoca, Romania.

2. Research Center for functional Genomics, Biomedicine and Translational Medicine, "Iuliu Hatieganu" University of Medicine and Pharmacy, Cluj-Napoca., Romania

3. Department of Pharmacology, Toxicology and Clinical Pharmacology, "Iuliu Haţieganu" University of Medicine and Pharmacy, Cluj-Napoca, Romania.

\begin{abstract}
Introduction. Vitamin D deficiency is a quite common pathology in European countries. Serum vitamin D levels are closely linked to physical fitness and lesser response to cardiac rehabilitation in various categories of patients with heart disease. Vitamin $\mathrm{D}$ is also involved in the function of heart cells via a vitamin D receptors found in cardiomyocytes. We present the case of a patient with vitamin D deficiency and symptomatic premature ventricular contractions (PVCs), in whom cardiac rehabilitation decreased PVC number, but association with vitamin D supplements led to a significant decrease in PVCs and complete disappearance of symptoms.
\end{abstract}

Keywords: cardiac rehabilitation, vitamin D, ventricular premature contractions, physical activity,

\section{Introduction}

Cardiac rehabilitation has been shown to be effective in patients with a history of myocardial infarction, aortocoronary bypass, percutaneous coronary interventions $(1,2)$ and different types of arrhythmia $(3,4)$. In addition to patients with arrhythmias, carriers of implantable devices such as pacemakers and defibrillators $(5,6)$ also benefit from cardiac rehabilitation. Current European and American guidelines recommend cardiac rehabilitation for all these categories of patients (7) as multiple studies demonstrated beneficial effects both in young adults and elderly $(8,9)$.

Inclusion in a cardiac rehabilitation program improves not only the individual's physical capacity, but also has been shown to decrease mortality by decreasing the number and severity of arrhythmic events (10).

We describe the case of a middle-aged patient who presented with ventricular premature contractions (PVCs) from the right ventricular outflow tract and who refused antiarrhythmic medication. Cardiac rehabilitation decreased the number of PVCs but vitamin D supplementation led to a significant decrease in PVCs and complete disappearance of symptoms.

\section{Case presentation}

We present the case of a 54-year-old patient who was discovered with PVCs on the occasion of a cardiological consultation for palpitations at rest and slight decrease of exercise capacity. The patient agreed with the publication of medical information on his case and signed the informed consent. PVC morphology suggested origin in the right ventricular outflow tract (RVOT) (Figure 1), and echocardiography was normal. During the exercise test performed up to $98 \%$ of the theoretical maximum heart rate, cardiac ischemia was excluded, and PVCs disappeared with reappearance at the end of exercise. Holter ECG detected 1350 PVCs/24 hours. As the patient refused any beta-blocker or antiarrhythmic medication, it was decided that he should perform cardiac rehabilitation for 2 months, with subjective monitoring of palpitations and PVCs during physical activity. For 2 months the patient ran 4 times / week, $10 \mathrm{~km} /$ day at a speed of 10 $\mathrm{km} /$ hour (Figure 2) on a flat track. After 2 months of physical training, palpitations decreased but still were present, and Holter ECG showed a slight decrease in the number of PVCs from 1350 to $1120 / 24$ hours. Vitamin D- 25-OH dosing showed a low value at $23 \mathrm{ng} / \mathrm{ml}$, therefore we decided to supplement vitamin D and continue cardiac rehabilitation with a Holter ECG after 30 days. Throughout this period, the patient received oral treatment with 5000 units / day vitamin D. Gradually the patient's symptoms disappeared, had no palpitations, and he continued at the same pace: $10 \mathrm{~km} /$ day within 1 hour, 4 times / week. At the end of the 4-week period, the vitamin D dosage showed a value of $32.4 \mathrm{ng} / \mathrm{ml}$ and Holter ECG 230 PVCs/24 hours (Figure 3). The patient continued the administration of vitamin D $2000 \mathrm{U} /$ day in the cold seasons without any palpitations at the end of 6 months follow-up.

\section{Discussion}

Due to the rapid advancement of therapeutic techniques in the recent years, patients with different types of rhythm disorders have been included in cardiac rehabilitation programs. From premature atrial contractions to atrial fibrillation and from premature ventricular contractions to ventricular tachycardia, patients with arrhythmias benefit from cardiac 
rehabilitation $(7,10)$. Prior to inclusion in a rehabilitation program, the patient should be evaluated both clinically (presence of heart failure signs) and by non-invasive tests: (ECG, Holter ECG, exercise testing, echocardiography), and sometimes invasive techniques in function of the complexity of the arrhythmia (coronary angiography, electrophysiological study). Physical activity has a double role in patients with cardiac arrhythmias: first it is a precipitator for rhythm disorder and second, it is also a factor of prevention and reduction of arrhythmia severity $(1,3,7,10)$.

The study of Boukhris et al (11). performed on 122 patients demonstrated that cardiac rehabilitation in diabetic patients improves repolarization parameters and PVC number evaluated by Holter ECG both in diabetic and non-diabetic patients. Additionally, PVC severity expressed by the Lown class, decreased at the end of the rehabilitation program in both diabetic and non-diabetic patients.

Vitamin D deficiency is a relatively common condition in European countries. A study conducted in France on a population of 2971 individuals $>18$ years of age, identified approximately $36.7 \%$ with vitamin D deficiency(12). Not only in France but also in other European countries, depending on the location and latitude, vitamin D deficiency is present, on average in about $13 \%$ of the general population (13).

In addition to its role in calcium and phosphorus homeostasis, vitamin D has other actions, some at the cardiac level by acting on the specific vitamin $\mathrm{D}$ receptor from cardiomyocytes.

Most studies have shown beneficial effects of vitamin D on exercise capacity (14). Tofanello et al. showed that low levels of vitamin D are associated with lower aerobic capacity and lower muscle strength in both men and women (15).

Following the association between exercise capacity and vitamin D levels in patients with heart failure, Boxer et al demonstrated that there is a close relationship between the two and the peak maximal oxygen consumption is closely related to vitamin D levels (16).

In our patient the PVC number slightly decreased with cardiac rehabilitation from 1350/24 hours to $1120 / 24$ hours during a 2-month period of time. However, only after the correction of vitamin D deficiency the decrease was significant, up to $230 / 24$ hours. We believe that vitamin D deficiency has an enormous importance in the occurrence of the so-called "benign" outflow tract PVCs. In this patient, PVCs occurred without an underlying structural cardiac disease and had an RVOT morphology. Vitamin D deficiency is bound to many manifestations that we consider idiopathic, such as fatigue, sweating, weakness, palpitations and has also been implicated in the occurence of PVCs $(17,18)$. Therefore we believe that further studies should be performed in patients with outflow tract PVCs, considered idiopathic, to determine the role of vitamin D deficiency in the occurrence of PVCs and if vitamin D supplementation has a role in the treatment of PVCs.

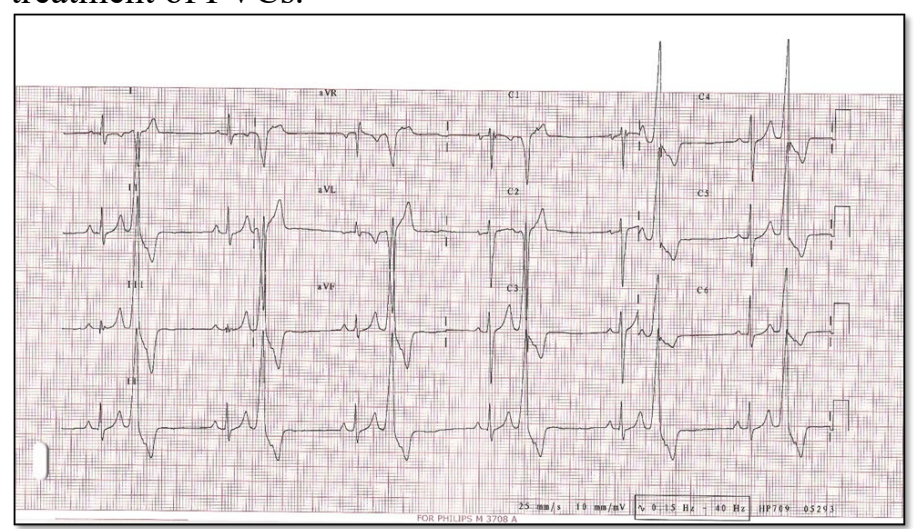

Figure 1: Twelve lead ECG shows sinus rhyhm with ventricular bigeminism. PVCs have a LBBB pattern with inferior axis and precordial transition in lead V3.

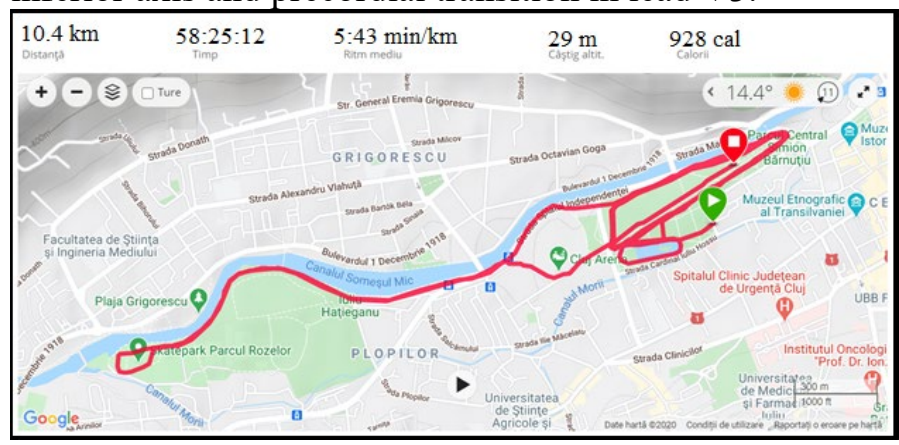

Figure 2. During physical training the patient was monitored with a Garmin Forerunner 235 Smartwatch which shows distance, speed and pace.

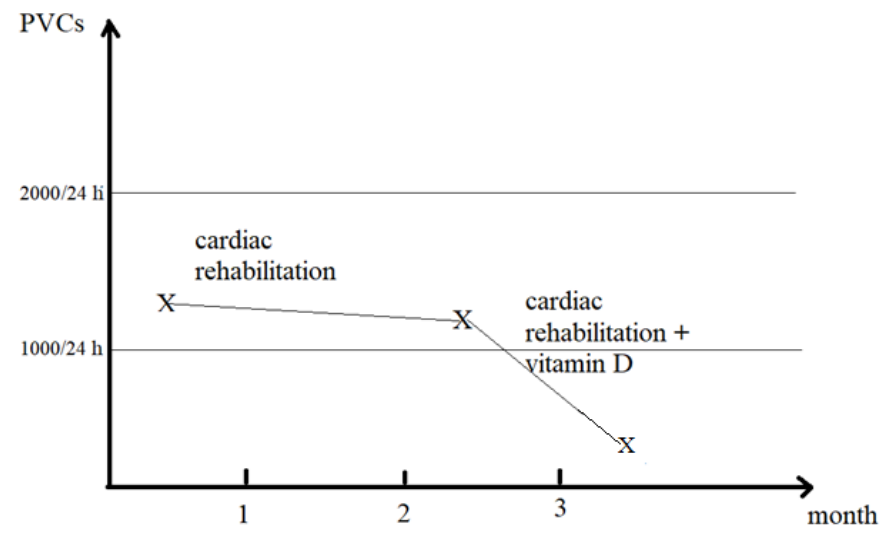

Figure 3. PVC burden decreases during 3 months period after application of cardiac rehabilitation or rehabilitation + vitamin D supplementation.

\section{Conclusion:}

Cardiac rehabilitation in patients with vitamin D deficiency and PVCs may be useful but not sufficient to increase exercise capacity. The addition of vitamin D can increase exercise capacity and lead to a significant decrease in the number of PVCs and disappearance of symptoms. 


\section{References:}

1. Malfatto G, Facchini M, Bragato R, Branzi G, Sala L, Leonetti G. Short and long term effects of exercise training on the tonic autonomic modulation of heart rate variability after myocardial infarction. Eur Heart J. 1996;17:532-538.

2. MocanM , Chiorescu R, Banc ON, Mocan B, Anton F, Stoia M, Farcas AD. Cardiac rehabilitation protocols outcome in frail patients undergoing transcatheter aortic valve implantation. Balneo Research Journal 2019:9:401-405.

3. Oldridge NB, Guyatt GG, Fischer ME, Rimm AA. Cardiac rehabilitation after myocardial infarction: combined experience of randomized clinical trials. J Am Med Assoc. 1988;260:945-950.

4. Moldovan O, Deak B, Bian A, Gurzau D, Frangu F, Martis A, Caloian B, Comsa H, Cismaru G, Pop D. How do I track Cardiac Rehabilitation in my patient with ischemic heart disease using Strava. Balneo Research Journal 2019:10:114-117.

5. Caloian B, Guşetu GN, Costea S, Comsa DH, Cismaru GL, Roşu RO, Pop D, Zdrenghea DT. The effect of cardiac pacemaker implantation on cardiac performance - the experience of a Cardiology Rehabilitation Department. Balneo Research Journal 2019:9:54-58.

6. Caloian B, Pop D, Guşetu G, Zdrenghea D. The role of cardiopulmonary exercise testing in the initial evaluation of patients wearing intracardiac devices submitted to cardiac rehabilitation. 2017:8:206-211.

7. O'Connor GT, Buring JE, Yusuf S, et al. An overview of randomized trial of rehabilitation with exercise after myocardial infarction. Circulation. 1989;80: 234-244.

8. Mocan M, Mocan B. Cardiac rehabilitation for older patients with cardiovascular pathology using robotic systems - A survey. Balneo Research Journal 2019:10:33-36.

9. Chan KA, Cismaru G, Istratoaie S, Puiu M, Gusetu G, Caloian B, Comsa H, Frangu F, Gurzau D, Martis A, Pop D, Zdrenghea D, Rosu R. Cardiac rehabilitation in elderly athletes. Analysis of elderly participation in Wizz Air Cluj-Napoca Marathon. Balneo Research Journal 2019:10:231-235.

10. Perk J, De Backer G, Gohlke H, et al. European Guidelines on Cardiovascular Disease Prevention in Clinical Practice (version 2012). The Fifth Joint Task Force of the European Society of Cardiology and other societies on cardiovascular disease prevention in clinical practice. Eur J PrevCardiol. 2012;19(4):585e667. 5. Thomas RJ, King M, Lui K, et al, AACVPR, ACC, AHA, American College of Chest Physicians, American College of Sports Medicine, American Physical Therapy Association, Canadian Association of Cardiac Rehabilitation,
European Association for Cardiovascular Prevention and Rehabilitation, Inter-American Heart Foundation, National Association of Clinical Nurse Specialists, Preventive Cardiovascular Nurses Association, Society of Thoracic Surgeons. AACVPR/ACC/ AHA 2007 performance measures on cardiac rehabilitation for referral to and delivery of cardiac rehabilitation/secondary prevention services endorsed by the American College of Chest Physicians, American College of Sports Medicine, American Physical Therapy Association, Canadian Association of Cardiac Rehabilitation, European Association for Cardiovascular Prevention and Rehabilitation, Inter-American Heart Foundation, National Association of Clinical Nurse Specialists, Preventive Cardiovascular Nurses Association, and the Society of Thoracic Surgeons. J Am Coll Cardiol. 2007;50:1400-1433.

11. MBoukhris M, Tomasello SD, Khanfir R. Impacts of cardiac rehabilitation on ventricular repolarization indexes and ventricular arrhythmias in patients affected by coronary artery disease and type 2 diabetes. Heart \& Lung 2015;44:199-204.

12. Salanave B., Vernay M., Szego E., Malon A., Deschamps V., Hercberg S. , et al.Physical activity patterns in the French 18-74-year-old population: French Nutrition and Health Survey (Etude Nationale Nutrition Santé, ENNS) 2006-2007Public Health Nutr, 15 (2012), pp. 2054-2059,

13. Cashman KD, Dowling KG, Škrabáková Z, et al. Vitamin D deficiency in Europe: pandemic?. Am J Clin Nutr. 2016;103(4):1033-1044. doi:10.3945/ajen.115.120873

14. Rejnmark L. Effects of vitamin D on muscle function and performance: a review of evidence from randomized controlled trials. Ther Adv Chronic Dis, 2 (2011), pp. 25-37

15. Toffanello E.D., Perissinotto E., Sergi G., Zambon S., Musacchio E., Maggi S., et al.

16. Vitamin D and physical performance in elderly subjects: the Pro.V.A. study PloS One, 7 (2012), p. e34950.

17. Boxer R.S., Dauser D.A., Walsh S.J., Hager, W.D. . Kenny A.M . The association between vitamin D and inflammation with the 6-minute walk and frailty in patients with heart failure J Am Geriatr Soc, 56 (2008), pp. 454-461

18. Kiuchi MG, Silva GR, Paz LMR et al. Influence of vitamin D levels on the treatment of premature ventricular complexes in patients with chronic kidney disease IJC MEthabolic and Endoctine 2017;14:53-58 